\title{
Os benefícios das terapias manuais na cefaleia tensional: uma revisão bibliográfica
}

\author{
The benefits of manual therapies in tension headache: a literature review \\ Los beneficios de las terapias manuales en la cefalea tenssional: una revisión de la literatura
}

Recebido: 20/09/2021 | Revisado: 25/09/2021 | Aceito: 26/09/2021 | Publicado: 27/09/2021

\author{
Sâmya Mércia Pinto Martins \\ ORCID: https://orcid.org/0000-0001-6611-1668 \\ Centro Universitário da Amazônia, Brasil \\ E-mail: samya.martins_@hotmail.com \\ Alexssandra Costa Melo \\ ORCID: https://orcid.org/0000-0002-9670-7304 \\ Centro Universitário da Amazônia, Brasil \\ E-mail: alemelo516@gmail.com \\ Indiara de Alencar \\ ORCID: https://orcid.org/0000-0003-3204-3278 \\ Universidade do Estado do Pará, Brasil \\ E-mail: indiara.alencarstm@gmail.com
}

\begin{abstract}
Resumo
A cefaleia do tipo tensional (CTT) é um quadro álgico presente no cotidiano de muitos cidadãos, ela é de origem multifatorial e está intimamente ligada a fatores intrínsecos como o emocional e extrínseco como o ambiente. O presente estudo se trata de uma revisão bibliográfica de caráter narrativo e abordagem analítica, entre o período de 2015 a 2020, buscou comprovar por meio de embasamentos científicos os benefícios que as técnicas de terapias manuais trazem para os pacientes com essa algia, desmitificando o seu tratamento apenas pelo o uso de fármacos. A atual pesquisa foi fundamentada em artigos retiradas das fontes de bases de dados Google Acadêmico, Scielo, Pubmed, PEDro e Jospt. Os critérios de inclusão foram artigos que apresentassem relação direta com o tema proposto, estudos que abordassem as terapias manuais utilizadas e que concluíssem com resultados positivos. Foram excluídos os artigos que não se enquadraram nos critérios de inclusão citados e que não apontassem pontos positivos da cefaleia com as terapias manuais. A partir da aplicação dos critérios foram selecionados 12 artigos condizentes com a temática do estudo. Após a análise e discussão dos dados, evidenciou-se através dos resultados os efeitos positivos que os métodos terapêuticos manuais trazem para cefaleia, foram abordadas as principais técnicas nesse estudo que totalizaram 13 terapias manuais. A pesquisa mostrou que os benefícios promovem não só a melhora na CTT, como também trouxe resultados benéficos na melhora da qualidade de vida, funcionalidade e qualidade do sono.
\end{abstract}

Palavras-chave: Terapias manuais; Cefaleia tensional; Dor.

\begin{abstract}
The tension type headache (CTT) is a pain present in the daily life of many citizens, it is of multifactorial origin and is closely linked to intrinsic factors such as the emotional and extrinsic as the environment. This study is a bibliographic review of narrative character and analytical approach, between the period 2015 to 2020, sought to prove through scientific foundations the benefits that manual therapy techniques bring to patients with this algia, Identifying your treatment only by using drugs. The current research was based on articles from the sources of Google Scholar, Scielo, Pubmed, Pedro and Jospt databases. The inclusion criteria were articles that presented a direct relationship with the proposed theme, studies that addressed the manual therapies used and concluded with positive results. Articles that did not meet the above inclusion criteria and that did not indicate positive aspects of headache with manual therapies were excluded. From the application of the criteria, 12 articles were selected in accordance with the theme of the study. After the analysis and discussion of the data, it was evidenced through the results the positive effects that the manual therapeutic methods bring to headache, the main techniques were addressed in this study that totaled 13 manual therapies. The research showed that the benefits promote not only the improvement in CTT, but also brought beneficial results in improving the quality of life, functionality and quality of sleep.
\end{abstract}

Keywords: Hand therapies; Tension headache; Pain.

\section{Resumen}

La cefalea de tipo tensional (CTT) es un cuadro álgico presente en el cotidiano de muchos ciudadanos, ella es de origen multifactorial y está íntimamente ligada a factores intrínsecos como el emocional y extrínseco como el ambiente. El presente estudio se trata de una revisión bibliográfica de carácter narrativo y enfoque analítico, entre el período de 2015 a 2020, buscó comprobar por medio de basamentos científicos los beneficios que las técnicas de terapias manuales traen para los pacientes con esa algia, desmitificando su tratamiento solo por el uso de fármacos. La 
investigación actual se basó en artículos extraídos de las fuentes de bases de datos Google Académico, Scielo, Pubmed, PEDro y Jospt. Los criterios de inclusión fueron artículos que presentaran relación directa con el tema propuesto, estudios que abordasen las terapias manuales utilizadas y que concluyeran con resultados positivos. Se excluyeron los artículos que no cumplían los criterios de inclusión citados y que no apuntaban puntos positivos de la cefalea con las terapias manuales. A partir de la aplicación de los criterios se seleccionaron 12 artículos acordes con la temática del estudio. Después del análisis y discusión de los datos, se evidenció a través de los resultados los efectos positivos que los métodos terapéuticos manuales traen para cefalea, se abordaron las principales técnicas en ese estudio que totalizaron 13 terapias manuales. La investigación ha demostrado que los beneficios no solo promueven la mejora en CTT, sino que también han traído resultados beneficiosos en la mejora de la calidad de vida, funcionalidad y calidad del sueño.

Palabras clave: Terapias manuales; Cefalea tensional; Dolor.

\section{Introdução}

A cefaleia do tipo tensional é caracterizada por episódios frequentes de quadros álgicos, tipicamente bilateral, com caráter de pressão ou aperto de intensidade de média à moderada, durando de minutos a dias, segundo a Classificação Internacional de Cefaleias. Conforme define a International Headache Society (2018), a cefaleia é segmentada em: primária, secundária, neuropatias dolorosas, dores faciais e dores de cabeça. Muitas vezes, essas manifestações advêm de pontos gatilhos, que são pontos irritáveis à palpação associados a um nódulo hipersensível (Araújo \& Almeida, 2009).

Tais pontos gatilhos podem ser divididos em dois tipos: ativos e latentes. Os ativos são mais dolorosos e apresentam hipersensibilidade, que causa dor no músculo ao fazer movimento, a palpação e tensão muscular, já os latentes são caracterizados por manifestarem dores menos intensas, não causa dor ao realizar atividades e são menos dolorosos durante a palpação (Santos, et al., 2010).

A cefaleia é uma manifestação frequente na prática clínica, com ocorrência de $90 \%$ durante a vida da população em geral. Estimativas mundiais indicam que a prevalência de enxaqueca, também denominada migrânea, é de aproximadamente $6 \%$ em homens e $15 \%$ a $18 \%$ em mulheres adultas, e a de cefaleia do tipo tensional de $90 \%$ em mulheres e $67 \%$ em homens. A cefaleia aparece como o motivo mais frequente de encaminhamentos a ambulatórios de neurologia (Ferreira, et al., 2006).

De acordo com a Sociedade Brasileira de Cefaleia (2002), a profilaxia e tratamento da cefaleia deve englobar os fármacos (betabloqueadores, antidepressivos, bloqueadores dos canais de cálcio, antagonistas de serotonina, antiepilépticos e miscelânea) e a fisioterapia pode ser utilizada em casos selecionados, tendo como tratamento complementar a acupuntura, porém ainda demostram poucos estudos.

As evidências científicas vêm mostrando ao decorrer do tempo os benefícios que as terapias manuais trazem para pessoas que tem a cefaleia tensional e nos últimos anos tem ganhado destaque, pois suas várias técnicas promovem a normalização do tecido membranoso por meio de micro movimentos do crânio, auxilia na drenagem venosa, diminuição da compressão nervosa e relaxamento dos tecidos moles diretamente relacionados ao quadro álgico (Hoffman \& Teodorosk, 2003).

Dentre as várias técnicas manuais utilizadas para o tratamento da cefaleia está a liberação miofascial que é compreendida por técnicas manuais em que a pressão é aplicada ao musculo e à fáscia muscular, com o objetivo de liberar a fáscia e facilitar as alterações de comprimento histológico, para avaliar alguns dos sintomas de restrição fascial (Carvalho, et al., 2017). Essa técnica também consiste na liberação da tensão dos músculos e das fáscias, aumentando a circulação local, reduz dor e espasmo, podendo propiciar alívio da CTT, através de técnicas fundamentais para a desativação de Pontos Gatilhos e seus sintomas (Souza, et al., 2015).

Diante disso a técnica osteopática denominada arcos botantes, consiste no afastamento do osso occipital e temporal para abertura do forame jugular (Ricard, 2002). Essa manobra é realizada devido ao forame ser um local de passagem de muitos nervos, que por sua vez se forem comprimidos causam a cefaleia, segundo Adams e Victor (1998). Por conseguinte, 
outra técnica eficaz para o controle álgico é a de Mulligan, se trata de uma forma de tratamento relativamente nova onde se aplica mobilizações articulares de baixa velocidade sem a manifestação de quadros álgicos que pode incluir um componente de movimento ativo (Hing, et al., 2019). Esta técnica proporciona o reposicionamento articular no nível cervical nos casos de diminuição da amplitude de movimento (ADM) na rotação das vértebras $\mathrm{C} 1$ e $\mathrm{C} 2$, a qual está frequentemente relacionada com a CTT (Mulligan, 2009).

Por conseguinte, a reeducação postural global exibe efeitos positivos nos quadros álgicos da CT também, pois se trata de uma técnica que utiliza posturas ativas e simultâneas, isométricas em posições excêntricas dos músculos da estática aplicadas em decoaptação articular progressiva, esse método desenvolve a consciência corporal e melhora a postura corporal (Rossi, et al., 2011 \& Soares, et al., 2016). A técnica de Jones ou terapia de liberação posicional, também tem se mostrado eficaz pois tem como base a aproximação das inserções musculares associada à compressão digital do ponto gatilho por 90 segundos, com objetivo de diminuir a tensão do músculo espasmódico. O princípio da técnica é palpar o ponto, desencadeando a dor e em seguida buscar a posição de relaxamento (chamada de silêncio neurológico sensorial), o que permite normalizar os tônus musculares (Simons, et al., 2007).

E, por conseguinte a técnica de inibição dos suboccipitais, a mesma tem como objetivo diminuir os espasmos e contrações musculares localizados na região occipital e produz a inibição dos tecidos moles de maneira a diminuir a compressão das estruturas cranianas que geram comorbidades como a cefaleia (Macedo, et al., 2007). Dessa forma, o presente estudo tem como objetivo analisar e apresentar a utilização das técnicas de terapias manuais nos quadros álgicos de cefaleia tensional, de forma a evidenciar ação profilática e melhora na qualidade de vida. A vista que ainda se encontram em escassez pesquisas que visem a abordagem dessas técnicas, a atual revisão visa contribuir para a concretização dos benefícios da fisioterapia manual.

\section{Metodologia}

Trata-se de uma revisão bibliográfica onde foram consultadas literaturas relativas ao assunto em estudo e artigos publicados em plataformas que possibilitaram que este trabalho tivesse embasamento cientifico para ser fundamentado. Segundo Marconi e Lakatos, 1992, a pesquisa bibliográfica é o levantamento de toda a bibliografia já publicada, em formas de revistas, livros e publicações, sendo que sua finalidade é fazer com que os pesquisadores entre em contato com todas as fontes de pesquisa sobre determinado assunto, sendo esta considerada o primeiro passo de toda pesquisa científica. A primore para a construção da revisão, foram selecionados artigos que tivessem relevância com a temática proposta, tendo como direcionamento a seguinte questão problema: De que maneira a aplicação das terapias manuais influenciam no tratamento de pacientes com cefaleia tensional.

Os critérios de inclusão foram artigos na língua inglesa e portuguesa dos anos de 2015 a 2020, que apresentassem relação direta com o tema proposto, estudos que tivessem amostras e dados como: idade, sexo, tipos de terapias manuais utilizados e resultados positivos. Foram excluídos os artigos que não estivessem disponíveis em versão completa ou de anos anteriores aos supracitados acima, artigos de revisão bibliográfica, resumos, estudos que não se enquadraram nos critérios de inclusão citados e que não apontassem pontos positivos da cefaleia com as terapias manuais.

Foram utilizadas as seguintes bases de dados: Public/Publisher MEDLINE (PubMed), Google Acadêmico, Scientific Eletronic Library Online (ScieLo), Physiotherapy Evidence Database (PEDro) e Journal of Orthopaedic \& Sports Physical Therapy (JOSPT) utilizando as palavras chaves: Cefaleia tensional, terapia manuais e dor, para busca dos artigos.

Utilizou-se uma tabela de autoria própria para a seleção e organização dos itens. Para melhor interpretação dos dados presentes no artigo, foram criadas colunas com títulos que facilitassem por meio dos critérios de inclusão e exclusão os estudos 
que seriam utilizados e os descartados, que foram: título do artigo, autor, amostra, sexo, idade, técnicas utilizadas e resultados.

Os estudos que não tivesse um desses critérios foram descartados, e os que não se enquadrasse no período de ano proposto.

\section{Resultados e discussão}

A partir da aplicação de critérios de inclusão e exclusão foram selecionados 12 artigos condizentes com a temática do estudo e que cumpriram com requisitos pré-estabelecidos para a análise e discussão dos resultados.

Tabela 1 - Resultados de estudos publicados dos anos de 2015 a 2020.

\begin{tabular}{|c|c|c|c|c|c|}
\hline Titulo & Autor & $\begin{array}{c}\text { Amostra e } \\
\text { sexo }\end{array}$ & Idade & Técnicas utilizadas & Resultados \\
\hline $\begin{array}{l}\text { Efeitos da técnica de } \\
\text { inibição dos músculos } \\
\text { suboccipitais na dor, } \\
\text { qualidade do sono e } \\
\text { incapacidade em pessoas } \\
\text { com cefaleia tensional. }\end{array}$ & $\begin{array}{c}\text { LIMA, K. V. et al. } \\
2020\end{array}$ & $\begin{array}{c}10 \\
\text { participantes, } \\
7 \text { mulheres e } \\
3 \text { homens }\end{array}$ & $\begin{array}{c}\text { Entre } 18 \mathrm{e} \\
40 \text { anos }\end{array}$ & $\begin{array}{l}\text { - Técnica de inibição dos } \\
\text { músculos suboccipitais }\end{array}$ & $\begin{array}{c}\text { A aplicação da técnica } \\
\text { ocasionou melhora } \\
\text { significativa na dor, } \\
\text { qualidade do sono e } \\
\text { funcionalidade em pessoas } \\
\text { com cefaleia tensional. }\end{array}$ \\
\hline $\begin{array}{l}\text { Os Efeitos da Liberação } \\
\text { Miofascial no Alívio da } \\
\text { Cefaleia Tensional: } \\
\text { Estudo Clínico, } \\
\text { Controlado e } \\
\text { randomizado }\end{array}$ & $\begin{array}{l}\text { BATISTA, A. V. } \\
2019\end{array}$ & $\begin{array}{l}11 \text { indivíduos } \\
\text { do sexo } \\
\text { feminino }\end{array}$ & $\begin{array}{c}\text { De } 18 \text { a } 30 \\
\text { anos }\end{array}$ & $\begin{array}{c}\text { Liberação Miofascial } \\
\text { - Inibição dos pontos } \\
\text { gatilhos } \\
\text { - Stretching dos músculos } \\
\text { esternocleidomastoideo, } \\
\text { escalenos e trapézio } \\
\text { superior. }\end{array}$ & $\begin{array}{l}\text { O tratamento ofertado teve } \\
\text { bons resultados sobre o } \\
\text { alívio da Cefaleia Tensional, } \\
\text { gerando não só o alivio da } \\
\text { dor como o alivio da tensão. }\end{array}$ \\
\hline $\begin{array}{c}\text { Efeitos da reeducação } \\
\text { postural global na } \\
\text { intensidade dos sintomas } \\
\text { álgicos e equilíbrio } \\
\text { postural em mulheres } \\
\text { jovens com cefaleia do } \\
\text { tipo tensional }\end{array}$ & KUNAST el al 2019 & $\begin{array}{l}6 \text { indivíduos } \\
\text { do sexo } \\
\text { feminino }\end{array}$ & $\begin{array}{l}\text { Com idade } \\
\text { média de } \\
28,1+-7,5 \\
\text { anos }\end{array}$ & Reeducação postural global & $\begin{array}{l}\text { Mostraram-se que com } 4 \\
\text { atendimentos através do } \\
\text { método RPG, houve } \\
\text { diminuição do quadro álgico } \\
\text { e redução do impacto em } \\
\text { mulheres jovens. }\end{array}$ \\
\hline $\begin{array}{l}\text { Influência da técnica de } \\
\text { Jones na cefaleia do tipo } \\
\text { tensional }\end{array}$ & $\begin{array}{c}\text { TEXEIRA, J. L. et } \\
\text { al. } 2018\end{array}$ & $\begin{array}{c}38 \\
\text { voluntários } \\
\text { sendo } 35 \text { do } \\
\text { sexo } \\
\text { feminino e } 3 \\
\text { do sexo } \\
\text { masculino }\end{array}$ & $\begin{array}{c}\text { De } 18 \text { a } 30 \\
\text { anos }\end{array}$ & $\begin{array}{c}\text { - Técnica de Jones } \\
\text { (mobilizações Articulares, } \\
\text { desativação de trigger } \\
\text { points musculares, liberação } \\
\text { posicional). }\end{array}$ & $\begin{array}{c}\text { A técnica de Jones } \\
\text { apresentou influência } \\
\text { positiva na melhora da } \\
\text { qualidade de vida do grupo } \\
\text { experimental, sendo } \\
\text { relevante no tratamento da } \\
\text { cefaleia do tipo tensional. }\end{array}$ \\
\hline $\begin{array}{l}\text { Eficácia da técnica arcos } \\
\text { botantes sobre indivíduos } \\
\text { com cefaleia primaria: } \\
\text { estudo randomizado } \\
\text { controlado }\end{array}$ & ALVES, V. S. 2018 & $\begin{array}{c}60 \\
\text { participantes } \\
\text { de ambos os } \\
\text { sexos }\end{array}$ & $\begin{array}{c}\text { De } 18 \text { a } 60 \\
\text { anos }\end{array}$ & -Técnica de arcos botantes & $\begin{array}{l}\text { Os resultados sugerem que a } \\
\text { técnica de arcos botantes } \\
\text { pode ser considerada um } \\
\text { procedimento clinicamente } \\
\text { válido para o tratamento de } \\
\text { pacientes com cefaleia } \\
\text { primária, em especial a } \\
\text { cefaleia tensional e a } \\
\text { enxaqueca. }\end{array}$ \\
\hline $\begin{array}{l}\text { Análise da mobilização } \\
\text { articular da cervical em } \\
\text { indivíduos com cefaleia } \\
\text { do tipo tensão }\end{array}$ & $\begin{array}{c}\text { KAROLCZAK, A. } \\
\text { P. B. et al. 2018 }\end{array}$ & $\begin{array}{c}15 \\
\text { voluntários } \\
\text { de ambos os } \\
\text { sexos }\end{array}$ & $\begin{array}{c}\text { Entre } 18 \text { e } \\
40 \text { anos }\end{array}$ & $\begin{array}{c}\text {-Técnicas de } \\
\text { reposicionamento articular } \\
\text { - SNAGS C1/C2 } \\
\text { - Auto SNAGS C1/C2 }\end{array}$ & $\begin{array}{l}\text { Houve como efeito imediato } \\
\text { a redução da dor e o } \\
\text { aumento da ADM cervical, } \\
\text { principalmente de rotação, o } \\
\text { que favoreceu o } \\
\text { reposicionamento articular } \\
(\mathrm{C} 1 / \mathrm{C} 2) \text { e a funcionalidade } \\
\text { de pacientes com crises de } \\
\text { CTT. }\end{array}$ \\
\hline $\begin{array}{l}\text { A eficácia da liberação } \\
\text { miofascial na região } \\
\text { cervical para o alívio da } \\
\text { cefaleia tensional }\end{array}$ & $\begin{array}{l}\text { SLEPICKA, B. C. O. } \\
\text { et al. } 2016\end{array}$ & $\begin{array}{c}20 \\
\text { participantes } \\
\text { sendo } 11 \\
\text { homens e } 9 \\
\text { mulheres }\end{array}$ & $\begin{array}{c}\text { Entre } 20 \text { e } \\
50 \text { anos }\end{array}$ & $\begin{array}{c}\text { - Liberação miofascial } \\
\text { - Tração cervical } \\
\text { - Acupuntura } \\
\text { - Mobilização vertebral }\end{array}$ & $\begin{array}{l}\text { O uso de terapias manuais } \\
\text { para cefaleias apresenta } \\
\text { evidências de melhoras, } \\
\text { sendo eficaz. Os resultados } \\
\text { são cada vez mais aceitos e } \\
\text { comprovados por estudos } \\
\text { científicos. }\end{array}$ \\
\hline
\end{tabular}




\begin{tabular}{|c|c|c|c|c|c|}
\hline & & & & $\begin{array}{l}\text {-Alongamentos } \\
\text { - Relaxamentos }\end{array}$ & \\
\hline $\begin{array}{l}\text { As técnicas de terapia } \\
\text { manual têm um efeito } \\
\text { positivo na qualidade da } \\
\text { vida das pessoas com } \\
\text { cefaleia do tipo } \\
\text { tensional? Um ensaio } \\
\text { clinico randomizado }\end{array}$ & $\begin{array}{l}\text { ESPÍ-LÓPEZ, G. V. } \\
\text { et al. } 2016\end{array}$ & $\begin{array}{l}62 \text { pacientes } \\
\text { mulheres }\end{array}$ & $\begin{array}{c}\text { Entre } 18 \text { e } \\
65 \text { anos }\end{array}$ & $\begin{array}{c}\text { - Pressão inibitória } \\
\text { suboccipital, manipulação } \\
\text { espinhal suboccipital e uma } \\
\text { combinação dos dois } \\
\text { tratamentos }\end{array}$ & $\begin{array}{c}\text { Todos os } 3 \text { tratamentos } \\
\text { foram eficazes na mudança } \\
\text { de diferentes dimensões da } \\
\text { qualidade de vida, porém o } \\
\text { tratamento combinado se } \\
\text { tornou mais viável para } \\
\text { melhora da qualidade de } \\
\text { vida }\end{array}$ \\
\hline $\begin{array}{c}\text { O efeito da manipulação } \\
\text { mais massagem } \\
\text { terapêutica contra } \\
\text { massagem terapêutica } \\
\text { isolada em pessoas com } \\
\text { cefaleia do tipo tensional. } \\
\text { Um ensaio clinico } \\
\text { randomizado controlado }\end{array}$ & $\begin{array}{l}\text { ESPÍ-LÓPEZ, G. V. } \\
\text { et al. } 2016\end{array}$ & $\begin{array}{c}105 \\
\text { indivíduos } \\
\text { sendo } 23 \\
\text { homens e } 82 \\
\text { mulheres. }\end{array}$ & $\begin{array}{l}\text { Entre } \\
18 \text { e } 65 \\
\text { anos }\end{array}$ & $\begin{array}{c}\text { - Grupo de tratamento } \\
\text { recebeu manipulação eixo- } \\
\text { occipital-atlas e tratamento } \\
\text { de tecidos moles por } \\
\text { massagem } \\
\text { - Grupo controle apenas } \\
\text { massagem }\end{array}$ & $\begin{array}{l}\text { As duas técnicas combinadas } \\
\text { trazem efeitos positivos na } \\
\text { intensidade da cefaleia }\end{array}$ \\
\hline $\begin{array}{l}\text { Efeitos da liberação } \\
\text { miofascial na qualidade e } \\
\text { frequência da dor em } \\
\text { mulheres com cefaleia do } \\
\text { tipo tensional induzidas } \\
\text { por pontos-gatilho }\end{array}$ & $\begin{array}{l}\text { SOUSA, R. C. et al. } \\
2015\end{array}$ & $\begin{array}{l}9 \text { pacientes } \\
\text { mulheres }\end{array}$ & $\begin{array}{c}35 \text { a } 58 \\
\text { anos }\end{array}$ & $\begin{array}{l}\text { - Liberação miofascial } \\
\text { - Compressão isquêmica de } \\
\text { Jhones } \\
\text { - Massagem miofascial } \\
\text { - Pompagem } \\
\text { - Alongamento miofascial }\end{array}$ & $\begin{array}{c}\text { Através das técnicas de } \\
\text { liberação miofascial, } \\
\text { proporcionou resultados } \\
\text { significativos no tratamento } \\
\text { da cefaleia do tipo tensional, } \\
\text { através da redução da } \\
\text { intensidade subjetiva global } \\
\text { da dor, alívio dos } \\
\text { componentes sensoriais e } \\
\text { afetivos. }\end{array}$ \\
\hline $\begin{array}{c}\text { Estudo comparativo entre } \\
\text { a terapia manual e a } \\
\text { TENS burst em pacientes } \\
\text { portadores de cefaleia } \\
\text { tensional }\end{array}$ & $\begin{array}{l}\text { FERNANDES, D. V. } \\
\text { et al. } 2015\end{array}$ & $\begin{array}{l}60 \text { pessoas, } \\
\text { sendo } \\
\text { selecionadas } \\
40 \text { devido os } \\
\text { critérios de } \\
\text { exclusão. } 35 \\
\text { do sexo } \\
\text { feminino e } 5 \\
\text { masculino }\end{array}$ & $\begin{array}{c}\text { De } 18 \text { a } 80 \\
\text { anos }\end{array}$ & $\begin{array}{c}\text {-Manobra cervical } \\
\text { - Liberação miofascial } \\
\text { - Pompagem global } \\
\text { - Mobilização dos tendões } \\
\text { ciático e mediano } \\
\text {-KABAT } \\
\text { - Mobilização cervical } \\
\text { - Técnicas de alongamento } \\
\text { - TENS burst }\end{array}$ & $\begin{array}{l}\text { Embora os tratamentos } \\
\text { tenham sido apresentados } \\
\text { com eficácia no que diz } \\
\text { respeito à intensidade da dor, } \\
\text { o uso de técnicas de terapia } \\
\text { manual confere ao sujeito } \\
\text { maior qualidade de vida, em } \\
\text { comparação com o TENS } \\
\text { Burst. }\end{array}$ \\
\hline $\begin{array}{c}\text { Massagem na cabeça e no } \\
\text { pescoço focada no ponto } \\
\text { de gatilho miofascial para } \\
\text { cefaleia tensional } \\
\text { recorrente: um ensaio } \\
\text { clinico randomizado e } \\
\text { controlado por placebo } \\
\end{array}$ & $\begin{array}{l}\text { MORASKA, A. F. et } \\
\text { al. } 2015\end{array}$ & $\begin{array}{c}56 \text { de ambos } \\
\text { os sexos }\end{array}$ & $\begin{array}{c}18 \text { a } 59 \\
\text { anos }\end{array}$ & $\begin{array}{l}\text { Massagem com liberação de } \\
\text { pontos de gatilho e placebo } \\
\text { através do ultrassom }\end{array}$ & $\begin{array}{l}\text { A massagem focada no } \\
\text { ponto de gatilho, pode ser } \\
\text { uma via de tratamento } \\
\text { importante para diminuição } \\
\text { da cefaleia tensional. }\end{array}$ \\
\hline
\end{tabular}

Fonte: Martins, Melo e Alencar (2021).

Embora na atualidade a cefaleia tensional (CT) costume ser tratada por muitos como um distúrbio álgico comum, o impacto negativo na qualidade vida dos cidadãos costuma ser bastante incidente. A presente revisão buscou elucidar e comprovar os efeitos positivos que as terapias manuais trazem para o tratamento da cefaleia do tipo tensional. A partir da coleta de dados de artigos selecionados foram retiradas as principais técnicas manuais que ofertassem melhores resultados para o quadro álgico proposto, dessa forma foi analisado qual o efeito cada uma proporcionou para seus participantes, onde foi observado melhora da dor, da funcionalidade, da qualidade do sono.

Em seu estudo Lima et al., 2020, realizou a execução da técnica de inibição dos músculos suboccipitais onde foi realizada em 10 indivíduos com idade igual ou superior a 18 anos, esse método que consiste em produzir um bloqueio dos 
tecidos moles, de forma a reduzir a compressão craniana, mostrou os resultados eficazes na redução da dor onde a queixa da persistência da dor foi relatada em menos de $1 \%$, melhora da qualidade do sono em $7 \%$ e da funcionalidade em $8 \%$ em pacientes que apresentaram cefaleia tensional.

Assim como a pesquisa anterior, Espí-lópez, et al., 2016, também mostra a eficácia da técnica de inibição do suboccipital, como também explana outras técnicas e comprova a beneficidade das terapias manuais no tratamento da cefaleia. Para sua pesquisa o mesmo usou uma amostra de 76 pessoas. Para execução das técnicas, os pacientes foram divididos em quatro grupos: pressão inibitória suboccipital; manipulação espinhal suboccipital; uma combinação dos dois tratamentos; e o grupo controle. Após um mês de acompanhamento, os resultados mostram maior eficácia da técnica de inibição do suboccipital e ao considerar a qualidade de vida o tratamento combinado das técnicas teve maiores resultados.

Em outro estudo desenvolvido por Espí-lópez, et al., 2016, observou a eficácia de uma combinação de técnica de manipulação e massagem, e apenas massagem, onde foram selecionados 105 participantes que estivessem sofrendo com CT durante mais de 3 meses, os indivíduos tinham idade entre 18 e 65 anos de ambos os sexos, sendo a maior prevalência no sexo feminino. Foram avaliadas a gravidade da cefaleia, mudanças na incapacidade, mudanças funcionais e emocionais. As medições foram feitas imediatamente após quatro semanas de tratamento. O grupo de tratamento foi submetido a manipulação do eixo occipital-atlas e tratamento dos tecidos moles através da massagem. E o grupo controle recebeu apenas massagem. Os participantes de ambos os grupos demonstraram significativa melhora quando focada na região suboccipital e cervical, na frequência, gravidade, emocional e funcional; os resultados foram mais prevalentes quando foi associada as duas técnicas confirmando assim a hipótese do estudo e os objetivos que foram estabelecidos.

Segundo Batista, 2019, tratamento baseado em liberação miofascial também promove benefícios no alivio cefaleia, ressalta que a incidência é mais prevalente em mulheres adultas com $90 \%$ dos casos, corroborando outros estudos que evidenciam que o gênero feminino é o mais acometido. Durante a pesquisa foi recrutado 13 pacientes com diagnóstico de cefaleia tensional e subdividido em 2 grupos (controle e intervenção), e a partir da execução da técnica em um comparativo com os grupos onde o de intervenção após receber o tratamento por meio da liberação teve melhora da dor de 7,5 para 2,6 segundo a EVA, e o grupo controle não se obteve diferença visto que não recebeu nenhuma intervenção terapêutica.

Concordando com pesquisa anterior, Sousa, et al., 2015, diz que a liberação miofascial possui técnicas fundamentais para a desativação de pontos de gatilhos, nos quais este, se ativam devido a fatores ergonômicos e por períodos prolongados, como estresse, ansiedade, privação de sono entre outros que podem ativar os pontos de gatilhos. Em seu estudo foram selecionadas 9 mulheres com faixa etária de 35 a 58 anos. As técnicas utilizadas foram: compressão isquêmica de Jones, massagem miofascial, pompagem e alongamento. Os resultados encontrados nesta pesquisa expõem o tratamento fisioterapêutico com técnicas de LM apresentou efeitos benéficos no tratamento de CT nas variáveis de estudo. As técnicas empregadas neste estudo têm se mostrado satisfatórias e resolutivas, na diminuição e frequência dos episódios de cefaleia.

Semelhante a esse estudo, Slepicka e colaboradores, 2016, também utilizaram a liberação miofascial, mas nesse caso o diferencial foi o foco em pontos gatilhos de dor. A amostra foi composta por 20 indivíduos de ambos os sexos com idade de 20 a 50 anos e com diagnóstico de cefaleia tensional episódica. A maior incidência de cefaleia episódica ocorreu no sexo masculino, porém o sexo feminino apresentou maior grau álgico no pré-teste. Os pacientes foram submetidos a técnica de liberação miofascial na região cervical, e ao final do estudo foi observado que a técnica utilizada foi efetiva no tratamento da CT, sendo que houve diminuição e frequência da dor, aumento da amplitude de movimento, diminuição no uso de fármacos e da melhora na qualidade de vida.

Concordando com mesmo raciocínio Moraska, et al., 2015 propôs um estudo de massagem focada no ponto de gatilho miofascial versus placebo através do ultrassom desafinado. O protocolo de massagem foi estruturado com os músculos que estão interligados com a cefaleia tensional produzindo assim maior eficácia nas primeiras sessões do que comparado com o 
grupo que recebeu a terapia por meio do placebo. Através da terapia aplicada os dados estatísticos mostram que houve uma redução na frequência dos sintomas durante a intervenção, e melhora na qualidade de vida e mais disposição dos voluntários. $\mathrm{O}$ tratamento através do placebo precisa ser mais explorado no ponto de vista do autor, sendo assim a terapia manual se faz uma alternativa de tratamento, segura e eficaz.

Além da diminuição do principal sintoma que é o álgico, as terapias manuais promovem muitos efeitos positivos para a população, porém apesar de seu vasto leque de benefícios, tais técnicas ainda são de pouco conhecimento para a sociedade. Segundo Teixeira \& Ruaro, 2018, a técnica de Jones é eficaz como recurso na melhora da dor, do grau de capacidade e da qualidade de vida, a técnica consiste em posicionar o paciente em decúbito dorsal e palpar a musculatura e promover uma pressão de 90 segundos. Sua amostra foi composta por 35 mulheres e 3 homens, sendo o grupo experimental composto por 18 indivíduos e 20 do grupo controle. O grupo submetido a técnica apresentou melhora nos níveis de capacidade.

Divergente ao estudo anterior Karolczak et al., 2018, deu ênfase na mobilização articular na cervical para melhora da cefaleia, notou-se que ao avaliar um grupo experimental de 15 voluntários com predomínio feminino, houveram melhoras significativas da dor, os métodos realizados foi a seleção de duas técnicas chamadas de SNAGS C1/C2 sendo passiva e auto SNAGS C1/C2 que são mobilizações ativa. O grupo de mobilização passiva teve redução da dor de 29,6\% e na ativa teve diminuição de $38,6 \%$.

Vale mencionar a reeducação postural global, como tratamento eficaz no combate a cefaleia, em estudo realizado por Kunast e colaboradores, 2019, foi aplicada a técnica com 6 mulheres, ressalta que após 4 atendimentos já mostraram melhora da dor, pois a postura inadequada como interiorização da cabeça e diminuição da força e resistência do músculos resultam em quadros álgicos, o que torna a técnica altamente eficaz tratando com alongamentos, descompressão articular e muscular com tração cervical, evitando compensações, alinhamento a postura e dessa forma promoveu o alivio da dor.

Comparando terapias manuais a eletroterapia, Fernandes, et al., 2015, em seu estudo realizou a aplicação do tens burst modulado por baixa frequência e a terapia manual através da manobra miofascial cervical e manual cranial aplicado, pompagem, mobilização dos tendões ciático e mediano, em seus voluntários. Em seus resultados, antes da intervenção o nível de dor em ambos os grupos era considerado crônica e com alto nível de exacerbação, e após o tratamento o grupo tratado com tens teve $50 \%$ de redução do quadro álgico ao toque muscular e vertebral. O grupo tratado com a terapia manual apresentou 100\% na redução dos sintomas álgicos, fazendo assim, com que no estudo a maior relevância para tratamento da cefaleia seja a utilização do mecanismo da terapia manual, pois ela atua de diferentes formas trazendo resultados benéficos para saúde e bemestar dos portadores da doença.

Tratamentos convencionais são os mais indicados, porém pouco utilizados, segundo Alves, 2018 a técnica de $\operatorname{arcos}$ botantes na cefaleia é um deles, em seu estudo utilizou uma amostra de 60 pacientes divididos em três subgrupos (intervenção, placebo e controle), para a pesquisa foi necessário que os participantes relatassem sentir dores de cabeça em média 10 vezes por mês durante no mínimo 3 meses. O grupo que participou da abordagem terapêutica recebeu tratamento por 3 meses em um total de 7 intervenções, as técnicas foram de abertura da sutura petrobasilar e occiptomastoidea pelos arcos botantes, foi exibido ao final da pesquisa as diferenças significativas entre os grupos na melhora da dor, onde mesmo a técnica sendo executada em um curto período de tempo e várias limitações se obteve uma melhora de 14,55 dias de dor em média para 8 dias no final.

\section{Conclusão}

A partir da análise criteriosa dos artigos foi possível evidenciar que as utilizações das técnicas de terapias manuais promovem efeitos benéficos na cefaleia tensional, onde foi elucidado que essas intervenções abordam diretamente a causa da dor, e promove efeitos positivos mais rápidos e com tempo de reabilitação menor, na maioria dos casos. Através da discussão 
dos artigos selecionados, as técnicas mostraram efeitos de forma imediata, obteve-se então uma resposta clara e contundente da eficácia terapêutica, de maneira a exibir os quadros de evoluções dos pacientes com a melhora dos quadros álgicos e queixas funcionais. A presente revisão mostrou o que o tratamento convencional traz bons resultados para os pacientes com cefaleia, de forma a reabilitar e promover melhor qualidade de vida. Foram selecionadas as principais técnicas terapêuticas para esse estudo que foram: Liberação Miofascial, Inibição do Suboccipital, Inibição de Pontos Gatilhos, Massagem Terapêutica, Reeducação Postural Global, Pompagem, Alongamento Miofascial, Mobilizações dos Tendões Ciático e Mediano, Mobilização Articular Cervical (Mulligan), Técnicas de Reposicionamento Articular (Snags C1/C2 e Auto Snags C1/C2), Técnica de Jones, Tração Cervical, Técnica de Arcos Botantes.

Contudo, ainda se faz necessário mais estudos sobre o presente tema, e maiores investimentos em pesquisas que abordem grupos experimentais, principalmente que busquem comprovar a efetividade de sua análise por meio de abordagens comparativas com grupos placebos e grupos com outras formas de tratamento como a eletroterapia, pois dessa forma há mais clareza dos maiores resultados que as técnicas manuais trazem na cefaleia. É preciso que haja melhor propagação do assunto para a população alvo, já que muitos ainda desconhecem a presente forma de intervenção tema desse estudo, e é essencial mais estudos com amostras em suas pesquisas, pois a maioria das publicações se tratava apenas de revisões.

\section{Agradecimentos}

Agradecemos a nossa orientadora, professora e amiga Indiara de Alencar por toda paciência e parceria na elaboração desse artigo, sem ela nada teria sido realizado e possível, obrigada por sempre se disponibilizar para nós ajudar e contribuir de forma contundente para nosso sucesso pessoal e profissional, nossa eterna gratidão.

\section{Referências}

Adams, R. D. \& Victor, M. (1998). Neurologia. (6a ed.), McGraw-Hill Interamericana.

Alves, V. S. \& Carvalho, P. (2018). Eficácia da técnica de arcos botantes sobre indivíduos com cefaleia primária: Estudo randomizado controlado. Portugal. Araújo, A. P. S., \& Almeida, C. A. (2009). Terapia manual versus acupuntura no tratamento da cefaleia: revisão de literatura. Revista Saúde e Pesquisa, 2(1), $107-113$.

Batista, A. V. (2019). Os Efeitos da Liberação Miofascial no Alivio da Cefaleia Tensional: Estudo Clínico, Controlado e Randomizado.

Carvalho, L. S. et al. (2017). Auto liberação miofascial x alongamento estático: efeitos sobre a flexibilidade de escolares. Revista CPAQV Centro de Pesquisas Avançadas em Qualidade de Vida-CPAQV Journal, 9(2).

Daniel, R. P. et al. (2017). Abordagem não-farmacológica na cefaleia do tipo tensional: efeitos da hidroterapia sobre a dor e a qualidade de vida. Rev Bras Neurol. 53(1):15-26.

Espí-López, G. V. et al. (2016). O Efeito da Manipulação Mais Massagem Terapêutica Contra Massagem Terapêutica Isoladas em Pessoas com Cefaleia do Tipo Tensional: Um Ensaio Clinico Randomizado Controlado.

Espí-López, G. V. et al. (2016). As Técnicas de Terapia Manual Têm Efeito Positivo na Qualidade de Vida das Pessoas com Cefaleia do tipo tensional? Um Ensaio Clínico Randomizado.

Fernandes, D. V. et al. (2015). Estudo Comparativo Entre a Terapia Manual e a TENS Burst em Pacientes Portadores de Cefaeias Tensionais. Fisioterapia em movimento, 28(2), 327-337.

Ferreira, P. \& Filho, M. (2006). Cefaléia do tipo tensional episódica: Avaliação clínica de 50 pacientes. Arquivos de Neuro-Psiquiatria, 64, $95-99$. https://doi.org/10.1590/S0004-282X2006000100019>.

Freitas, J. P. et al. (2008). O efeito do método PRT na diminuição da dor em pacientes com pontos-gatilhos no músculo trapézio superior. XII INIC / VIII EPG - UNIVAP.

Galdino, G. S. et al. (2007). Cefaleias primárias: Abordagem diagnóstica por médicos não-neurologistas. Arquivos de Neuro-psiquiatria ;65(3-A):681-684.

Headache Classification Commitee Of The International Headache Society. (2013). The International Classification of Headache Disorders, 3rd edition (beta version). Cephalalgia. (33), 629-808.

Headache Classification Commitee Of The International Headache Society. (IHS). (2018). The International Classification of Headache Disorders, 3rd edition, Cephalalgia. 38(1), 1-211. 
Hing, W. et al. (2019). O conceito Mulligan de terapia manual (2a ed.),

Hoffmann, J. \& Teodorosk, R. (2003). A eficácia da pompage, na coluna cervical, no tratamento da cefaléia do tipo tensional. Terapia manual, 2(2), 56-60.

Karolczak, A. P. B. et al. (2018). Analise da Mobilização Articular da Cervical em Indivíduos com Cefaleia do Tipo Tensão. Revista FisiSenectus, 6(2), 16-25.

Kunast, D. C. D. et al. (2019). Efeitos da Reeducação Postural Global na Intensidade dos Sintomas Álgicos e Equilíbrio Postural em Mulheres Jovens com Cefaleia do Tipo Tensional. Fisioterapia Brasil, 20(6).

Lima, K. V. et al. (2020). Efeitos da técnica de inibição dos músculos occipitais na dor, qualidade do sono e incapacidade em pessoas com cefaleia tensional. Revista Fisioter Bras. https://doi.org/10.33233/fb.v21i2.4030.

Macedo, C. S. G. et al. (2007). Eficácia de terapia manual craniana em mulheres com cefaléia. Rev Fisioter Pesqui 14:14-20. https://doi.org/10.1590/fpusp.v14i2.75781.

Marconi, M. A. \& Lakatos, E. M. (1992). Metologia do trabalho cientifico. Editora Atlas, (4a ed.), 43 e 44

Medeiros, A. A. et al. (2012). A eficácia da fisioterapia manual na cefaléia tensional: uma revisão sistemática. Ter Man.10(47):100-4. 10.9736/TerMan.v10. n 47.73 .

Moraska, A. F. et al. (2015) Massagem na Cabeça e Pescoço Focada no Ponto Gatilho Miofascial para Cefaleia do Tipo Tensional Recorrente: Um Ensaio Clinico Randomizado e Controlado por Placebos.

Mulligan, B. R. (2009). Terapia manual: Técnicas NAGS - SNAGS - MWM e suas variantes. (5a ed.), Premier.

Organização Mundial da Saúde. (2011). Levantamento da incidência de cefaleia no brasil. http://www.who.int/healththinfo/statistics/bod_migraine.pdf.

Ricard, F. (2002). Tratado de osteopatia craneal, análisis ortodôntico, diagnóstico y tratamiento manual de los síndromes craneomandibulares. Panamericana. 1. ed. Madrid.

Rossi, L. P. et al. (2011). Efeito agudo da técnica de reeducação postural global na postura de mulheres com encurtamento da cadeia muscular anterior. Fisioter Mov 24:255-63. https://doi.org/10.1590/S0103-51502011000200007.

Santos, L. A., Sandin, G. R., \& Sakae, T. M. (2010). Associação de cefaleia e ansiedade em estudantes de Medicina de uma universidade do sul de Santa Catarina. Revista da AMRIGS, 54 (3): 288-293.

Sanvito, W. L. \& Monzillo, P. H. (1997). Cefaléias primárias: aspectos clínicos e terapêuticos. Simpósio Cefaléia; p.437-48. Cap.III.

Simons, D. G. \& Travell, J. G. (2005). Dor e disfunção miofascial, manual dos pontos- gatilho. (2a ed.), Artmed.

Soares, P. et al. (2016). Efeitos do Programa Escola de Postura e Reeducação Postural Global sobre a amplitude de movimento e níveis de dor em pacientes com lombalgia crônica. Rev Andaluza Med del Deport 9:23-28. https://doi.org/10.1016/j.ramd.2015.02.005.

Souza, J. A. (2002). Recomendações para o tratamento profilático da migrânea: Consenso da Sociedade Brasileira de Cefaleia. Arq Neuropsiquiatr ;60(1):15969. https://doi.org/10.1590/S0004-282X2002000100030.

Sousa, R. C. et al. (2015). Efeitos da liberação miofascial na qualidade e frequência da dor em mulheres com cefaleia do tipo tensional induzida por pontosgatilho. Fisioterapia Brasil, 16(3), 231-235. https://doi.org/10.1590/S0004-282X2002000100030.

Subcomitê de Classificação das Cefaleias da Sociedade Internacional de Cefaleia. (2006). Classificação Internacional das Cefaleias $2^{a}$ edição. ICHDII. Tradução da Sociedade Brasileira de Cefaleia com autorização da Sociedade Internacional de Cefaleia. São Paulo: Editora Farma. 62-73.

Slepicka, B. C. O. et al. (2016). A eficácia da Liberação Miofascial na Região Cervical para o Alivio da Cefaleia Tensional.

Stovner, L. J. et al. (2007). The global burden of headache: a documentation of headache prevalence and disability worldwide. Cephalalgia. 2007; 27:193-210.

Texeira, J. L \& Ruaro, J.A. (2018). Influência da técnica de Jones na cefaleia do tipo tensional. Revista inspirar, 17(3).

Vercelino, R. \& Carvalho, F. (2010). Evidências da acupuntura no tratamento da cefaleia. Rev Dor. São Paulo, out-dez;11(4):323-328. 